\title{
A NOTE ON CONTINUATION PROBLEMS
}

\author{
by RITA NUGARI
}

(Received 4 December, 1984)

Recently M. Martelli [6] and M. Furi and M. P. Pera [1] proved some interesting results about the existence and the global topological structure of connected sets of solutions to problems of the form:

$$
L x=N(\lambda, x)
$$

with $L: E \rightarrow F$ a bounded linear Fredholm operator of index zero (where $E, F$ are real Banach spaces), and $N: \mathbb{R} \times E \rightarrow F$ a nonlinear map satisfying suitable conditions.

While the existence of solution sets for this kind of problem follows from the Leray-Schauder continuation principle, it is our aim to show in this note that their global topological structure can be obtained as a consequence of the theory developed by J. Ize, I. Massabò, J. Pejsachowicz and A. Vignoli in $[\mathbf{3}, \mathbf{4}]$ about parameter dependent compact vector fields in Banach spaces.

Finally we give an example of a boundary value problem which has a connected subset of solutions "looking like" a loop.

1. Let $E, F$ be Banach spaces over $\mathbb{R}$ and let $U$ be an open subset of $\mathbb{R} \times E$. For $\lambda \in \mathbb{R}$, let $U_{\lambda}$ denote the fibre of $U$ at $\lambda$, i.e.

$$
U_{\lambda}=\{x \in E /(\lambda, x) \in U\} .
$$

We consider the problem

$$
L x=N(\lambda, x), \quad(\lambda, x) \in U,
$$

where (i) $L: E \rightarrow F$ is a linear bounded Fredholm operator of index zero, namely, if $\operatorname{Ker} L$ and $\operatorname{Im} L$ respectively denote the kernel and the image of $L$ then $\operatorname{Im} L$ is a closed subspace of $F$ and

$$
\operatorname{dim} \operatorname{Ker} L=\operatorname{codim} \operatorname{Im} L<+\infty \text {; }
$$

(ii) $N: \bar{U} \rightarrow F$ is a continuous map which is bounded (i.e. maps bounded sets onto bounded sets) and satisfies the following assumptions:

(N1) $N$ is $L$-compact (in the sense specified below);

(N2) there exists $\lambda_{0} \in \mathbb{R}$ such that $N\left(\lambda_{0}, x\right)=0$ for all $x \in \bar{U}_{\lambda_{0}}$, moreover $N$ has a continuous partial derivative $N_{\lambda}^{\prime}\left(\lambda_{0}, x\right)$ with respect to $\lambda$ at $\lambda_{0}$ with

$$
N_{\lambda}^{\prime}\left(\lambda_{0}, x_{0}\right) \neq 0 \text { for some } x_{0} \in \bar{U}_{\lambda_{0}} \text {. }
$$

Since $N\left(\lambda_{0}, x\right)=0$ for all $x \in \bar{U}_{\lambda_{0}}$, any $(\lambda, x)$ with $\lambda=\lambda_{0}$ and $x \in \operatorname{Ker} L \cap U_{\lambda_{0}}$ trivially solves (1.1), and we shall look for nontrivial solutions of this problem (a solution will always be seen as a pair $(\lambda, x) \in U)$.

Glasgow Math. J. 28 (1986) 55-61. 

write:

In the sequel we shall take $\lambda_{0}=0$ for simplicity of notation. Then due to (N2) we can

$$
N(\lambda, x)=\lambda A(x)+R(\lambda, x),
$$

where $A(x)=N_{\lambda}^{\prime}(0, x)$ (on identifying the linear map $N^{\prime}(0, x): \mathbb{R} \rightarrow F$ with the corresponding vector of $F$ ) is nontrivial and $R$ satisfies $R(\lambda, x) / \lambda \rightarrow 0$ as $\lambda \rightarrow 0$.

We shall now use the classical Liapunov-Schmidt procedure to reduce the problem (1.1) to an equation of the form:

$$
x=H(\lambda, x), \quad(\lambda, x) \in U,
$$

with $H: \bar{U} \rightarrow E$ a suitable compact map. Indeed let $E_{1}, F_{1}$ be closed subspaces of $E, F$ respectively such that $E=\operatorname{Ker} L \oplus E_{1}, F=\operatorname{Im} L \oplus F_{1}$, and let $P$ (resp. $Q$ ) denote the corresponding linear projection of $F\left(\right.$ resp. $E$ ) onto $F_{1}(\operatorname{resp} . \operatorname{Ker} L)$. Then $\left.L\right|_{E_{1}}$ is a one to one mapping of $E_{1}$ onto $\operatorname{Im} L$ and we shall set

$$
K=\left(\left.L\right|_{E_{1}}\right)^{-1}: \operatorname{Im} L \rightarrow E_{1} .
$$

Then the requirement (N1) of $L$-compactness on $N$ means that the map $K(I-P) N: \bar{U} \rightarrow$ $E_{1}$ is compact (see e.g. Gaines-Mawhin [2]).

Since $x=Q x+(I-Q) x$ and $L Q x=0,(1.1)$ is equivalent to

$$
L(I-Q) x=\lambda A(x)+R(\lambda, x)
$$

which, in turn, is equivalent to the following system obtained by using the complementary projections $P, I-P$ in $F$ :

$$
\left.\begin{array}{rl}
0 & =\lambda P A(x)+P R(\lambda, x), \\
(I-Q) x & =\lambda K(I-P) A(x)+K(I-P) R(\lambda, x) .
\end{array}\right\}
$$

Next define a continuous map $\hat{R}: \bar{U} \rightarrow F$ by

$$
\hat{R}(\lambda, x)= \begin{cases}\lambda^{-1} R(\lambda, x) & \text { if } \lambda \neq 0, \\ 0 & \text { if } \lambda=0\end{cases}
$$

and consider the modified system

$$
\left.\begin{array}{l}
0=P A(x)+P \hat{\bar{R}}(\lambda, x), \\
(I-Q) x=\lambda K(I-P) A(x)+K(I-P) R(\lambda, x) .
\end{array}\right\}
$$

Observe that the solution set of $\left(1.2^{\prime}\right)$ is contained in the solution set of (1.2): more precisely, the two systems are equivalent for $\lambda \neq 0$; for $\lambda=0$ the pair $(0, x)$ solves (1.2) iff $x \in \operatorname{Ker} L \cap U_{0}$ while it solves $\left(1.2^{\prime}\right)$ iff

$$
x \in \operatorname{Ker} L \cap U_{0} \text { and } A(x) \in \operatorname{Im} L .
$$

Let now $J$ be an isomorphism of $F_{1}$ onto $\operatorname{Ker} L$; if we use $J$ in the first equation of $\left(1.2^{\prime}\right)$ and sum the two equations, we see that $\left(1.2^{\prime}\right)$ is equivalent to the problem

$$
G(\lambda, x)=x-H(\lambda, x)=0,
$$


where

$$
H(\lambda, x)=Q x+J P A(x)+J P \hat{R}(\lambda, x)+\lambda K(I-P) A(x)+K(I-P) R(\lambda, x) .
$$

Notice that our assumptions imply that $H$ is a compact map. In this situation we can apply the results of [3] about the existence and topological properties of connected subsets of solutions of (1.3). In particular, the following result is an immediate consequence of Theorem 4.1 in [3]. We shall set, here and henceforth,

$$
G_{0}(x)=G(0, x)=x-(Q x+J P A(x)) .
$$

THEOREM 1. Let $G_{0}$ be as above and assume that there exists an open bounded subset $U^{\prime} \subset U_{0}$ such that the degree $\operatorname{deg}\left(G_{0}, U^{\prime}, 0\right)$ is defined (i.e. $G_{0}^{-1}(0) \cap U^{\prime}$ is compact) and nonzero. Then problem (1.1) has a closed connected set of solutions $\Sigma$,

$$
\Sigma \subset\{(\lambda, x) \in U: \lambda \neq 0\} \cup\left\{\{0\} \times U^{\prime}\right\},
$$

which meets $\{0\} \times U^{\prime}$ and satisfies one of the following: either $\Sigma$ is unbounded, or $\bar{\Sigma} \cap \partial U \neq \varnothing$, or $\bar{\Sigma}$ meets again the fibre $\lambda=0$ outside $U^{\prime}$. Moreover, any point $x$ at which $\bar{\Sigma}$ meets the fibre $\lambda=0$ satisfies

$$
x \in \operatorname{Ker} L \text { and } A(x) \in \operatorname{Im} L .
$$

Remark. Clearly the result of Theorem 1 holds true when (N2) is replaced by

$\left(\mathrm{N} 2^{\prime}\right)$ there exists an integer $s \geqq 1$ such that $N$ is $s$ times continuously differentiable with respect to $\lambda$ at $\lambda_{0}$ and

$$
\begin{gathered}
N\left(\lambda_{0}, x\right)=\ldots=\partial^{s-1} N\left(\lambda_{0}, x\right) / \partial \lambda^{s-1}=0 \quad \forall x \in \bar{U}_{\lambda_{0}}, \\
A(x):=\partial^{s} N\left(\lambda_{0}, x\right) / \partial \lambda^{s} \neq 0 \text { for some } \quad x \in \bar{U}_{\lambda_{0} .}
\end{gathered}
$$

2. Let us now show how Theorem 1 describes in a unified way the results contained in Furi-Pera [1] and Martelli [6].

Corollary 1 [1]. Assume that the operators $L, N$ are as described in $\$ 1$ and moreover (N2') holds. Define $v: \operatorname{Ker} L \cap U^{\prime} \rightarrow F_{1}$ by $v=P A i$, where $i$ is the inclusion map of $\operatorname{Ker} L \cap \bar{U}^{\prime}$ into $\bar{U}^{\prime}$. Assume that $\operatorname{deg}\left(v, \operatorname{Ker} L \cap U^{\prime}, 0\right)$ is defined and nonzero. Then the conclusion of Theorem 1 holds true.

Proof. Recall that

$$
G_{0}(x)=x-(Q x+J P A(x))
$$

and observe that $Q+J P A$ maps $E$ into the finite dimensional subspace $\operatorname{Ker} L$; therefore, by well-known properties of the Leray-Schauder degree (see e.g. Lloyd [5]),

$$
\operatorname{deg}\left(G_{0}, U^{\prime}, 0\right)=\operatorname{deg}\left(\left.G_{0}\right|_{\text {Ker } L}, \operatorname{Ker} L \cap U^{\prime}, 0\right)
$$

and, since $x=Q x$ on $\operatorname{Ker} L$,

$$
\begin{aligned}
\operatorname{deg}\left(G_{0}, U^{\prime}, 0\right) & =\operatorname{deg}\left(-J P A, \operatorname{Ker} L \cap U^{\prime}, 0\right) \\
& = \pm \operatorname{deg}\left(v, \operatorname{Ker} L \cap U^{\prime}, 0\right)
\end{aligned}
$$

because $J: F_{1} \rightarrow \operatorname{Ker} L$ is an isomorphism. The result now follows. 
REMARK. In [1], $U^{\prime}$ is taken to be the whole fibre $U_{0}$ : evidently, this particular case rules out the third possibility in Theorem 1, i.e. that $\Sigma$ "curls back" to the fibre $\lambda=0$ outside $U^{\prime}$.

On the other hand, in [1] the result is improved to show that each one of the sets $\Sigma^{+}$, $\Sigma^{-}$defined by

$$
\Sigma^{+}=\{(\lambda, x) \in \Sigma / \lambda>0\}, \quad \Sigma^{-}=\{(\lambda, x) \in \Sigma / \lambda<0\}
$$

is either unbounded or meets $\partial U$. However this can also be obtained as an easy consequence of Proposition 3.2 in [3], which in this context states that both $\Sigma^{+} \cap \partial V$ and $\Sigma^{-} \cap \partial V$ are nonempty whenever $V$ is an open bounded set such that $\Sigma_{0} \subset V \subset \bar{V} \subset U$, where

$$
\Sigma_{0}=\{(\lambda, x) \in \Sigma / \lambda=0\} .
$$

Corollary 2 [6]. Assume that $N(\lambda, x)=\lambda A(x)$ and that the following conditions are satisfied:

(i) there exists $x_{0} \in \operatorname{Ker} L$ such that $A\left(x_{0}\right) \in \operatorname{Im} L$;

(ii) $A$ is of class $C^{1}$ on some neighborhood $\stackrel{A}{U}$ of $x_{0}$;

(iii) $\left.P A^{\prime}\left(x_{0}\right)\right|_{\operatorname{Ker} L}: \operatorname{Ker} L \rightarrow F_{1}$ is an isomorphism.

Then the conclusion of Theorem 1 holds true and moreover

$$
\Sigma \subset\{(\lambda, x) \in U: \lambda \neq 0\} \cup\left\{\left(0, x_{0}\right)\right\} .
$$

Proof. By (i),

$$
G_{0}\left(x_{0}\right)=x_{0}-Q x_{0}-J P N\left(x_{0}\right)=0
$$

and the Fréchet derivative of $G_{0}$ at $x_{0}$,

$$
G_{0}^{\prime}\left(x_{0}\right)=I-Q-J P A^{\prime}\left(x_{0}\right),
$$

is an isomorphism, for it is injective by (iii) and of the form $I-K, K$ linear and compact ( $I$ is the identity map in $E$ ). Therefore, by the inverse mapping theorem, $G_{0}$ is a diffeomorphism of an open neighborhood $U^{\prime} \subset \hat{U}$ of $x_{0}$ onto an open neighborhood $V$ of $G_{0}\left(x_{0}\right)=0$, which implies that the Leray-Schauder degree $\operatorname{deg}\left(G_{0}, U^{\prime}, 0\right)$ is defined and nonzero.

The inclusion (2.1) is an obvious consequence of the fact that, in this case,

$$
\Sigma \cap\left\{\{0\} \times U^{\prime}\right\}=\left\{\left(0, x_{0}\right)\right\} .
$$

3. We end this note with a simple example in which the third case in Theorem 1 occurs and, moreover, the solution set contains a "loop" around a point $\left(\lambda_{0}, x_{0}\right)$, i.e. a connected subset containing $\left(\lambda_{0}, x_{0}\right)$ which is locally, but not globally, disconnected on removing the point $\left(\lambda_{0}, x_{0}\right)$. More general boundary value problems will be considered in a forthcoming paper.

Consider the problem

$$
\left.\begin{array}{c}
\dot{x}=\lambda\left(x^{2}-\left(1-\lambda^{2}\right) h\right) \\
x(0)=x(T)
\end{array}\right\}
$$


with $\lambda \in \mathbb{R}, T>0$ and $h$ a continuous function on $[0, T]$ with positive mean value

$$
\bar{h}=(1 / T) \int_{0}^{T} h(t) d t>0 .
$$

Let

$$
\begin{gathered}
E=\left\{x \in C^{1}([0, T]) / x(0)=x(T)\right\}, \\
F=C^{0}([0, T])
\end{gathered}
$$

with the usual norms $\|x\|_{F}=\sup \{|x(t)|: t \in[0, T]\},\|x\|_{E}=\|x\|_{F}+\|\dot{x}\|_{F}$. Define $L: E \rightarrow F$ by $L x=\dot{x}$; then $L$ is a bounded linear Fredholm operator of index zero and

$\operatorname{Ker} L=\{x \in E / x$ is a constant function $\}$,

$$
\operatorname{Im} L=\left\{y \in F / \int_{0}^{T} y(t) d t=0\right\} .
$$

Let $F_{1}$ be the subspace of constant functions in $F$ and $P: F \rightarrow F_{1}$ be defined by

$$
P y=(1 / T) \int_{0}^{T} y(t) d t .
$$

Let moreover $N: \mathbb{R} \times E \rightarrow F$ be defined by $N(\lambda, x)=\lambda\left(x^{2}-\left(1-\lambda^{2}\right) h\right)$; then $N$ is compact (and therefore $L$-compact) and satisfies (N2) with $\lambda_{0}=0$,

$$
A(x)=x^{2}-h \text { and } R(\lambda, x)=\lambda^{3} h .
$$

We shall now find some easy a priori bounds for the solutions of (3.1) with $\lambda \neq 0$. First observe that, if $(\lambda, x)$ solves (3.1) then, on integrating both sides and using the boundary conditions $x(0)=x(T)$, we get

therefore, if $\lambda \neq 0$,

$$
0=\lambda \int_{0}^{T}\left[x^{2}(t)-\left(1-\lambda^{2}\right) h(t)\right] d t
$$

$$
\int_{0}^{T} x^{2}(t) d t=\left(1-\lambda^{2}\right) \int_{0}^{T} h(t) d t
$$

which shows that $|\lambda| \leqq 1$, due to the assumption on $h$.

To find a priori bounds for $x$, let $t_{0} \in[0, T]$ be such that $x\left(t_{0}\right)=\max \{x(t): t \in[0, T]\}$. If $t_{0} \in(0, T)$ then $\dot{x}\left(t_{0}\right)=0$ and so, if $\lambda \neq 0, x^{2}\left(t_{0}\right)=\left(1-\lambda^{2}\right) h\left(t_{0}\right)$ which implies $x\left(t_{0}\right) \leqq\|h\|_{F}^{1 / 2}$. If the maximum is achieved at the endpoints then $\dot{x}(0) \dot{x}(T) \leqq 0$, i.e.

$$
\left[x^{2}(0)-\left(1-\lambda^{2}\right) h(0)\right]\left[x^{2}(T)-\left(1-\lambda^{2}\right) h(T)\right] \leqq 0
$$

which implies $x(0)=x(T) \leqq\|h\|_{F}^{1 / 2}$. We can bound $x(t)$ from below in a similar way and conclude that

$$
\|x\|_{F} \leqq\|h\|_{F}^{1 / 2} .
$$

Using again the equation, we have

$$
|\dot{x}(t)| \leqq|\lambda|\left(x^{2}(t)+\left(1-\lambda^{2}\right) h\right) \leqq\|h\|_{F}+\|h\|_{F}=2\|h\|_{F} ;
$$


whence finally

$$
\|x\|_{E}=\|x\|_{F}-\|\dot{x}\|_{F} \leqq 2\|h\|_{F}+\|h\|_{F}^{1 / 2} .
$$

In order to apply Theorem 1, we need to find an open bounded subset $U^{\prime}$ of $E$ such that $\operatorname{deg}\left(G_{0}, U^{\prime}, 0\right)$ is defined and nonzero, where $G$ and $G_{0}$ are as in (1.3), (1.4). As already remarked, $x \in E$ solves $G_{0}(x)=0$ iff $x \in \operatorname{Ker} L$ and $A(x) \in \operatorname{Im} L$; therefore in our case

$$
\begin{gathered}
x=\text { const }=\bar{x}, \\
0=P\left(x^{2}-h\right)=\bar{x}^{2}-P h=\bar{x}^{2}-\bar{h} .
\end{gathered}
$$

Hence $G_{0}^{-1}(0)=\left\{x^{+}, x^{-}\right\}$with $x^{ \pm}= \pm \alpha$, where $\alpha=\bar{h}^{1 / 2}$. Therefore, if $B^{+}$denotes a sufficiently small ball in $E$ around $x^{+}$, the Leray-Schauder degree $\operatorname{deg}\left(G_{0}, B^{+}, 0\right)$ is defined. As in the proof of Corollary 1 ,

$$
\operatorname{deg}\left(G_{0}, B^{+}, 0\right)=\operatorname{deg}\left(-\left.J P A\right|_{\text {Ker } L}, \operatorname{Ker} L \cap B^{+}, 0\right)
$$

and $J P A(x)=J P\left(x^{2}-h\right)=J\left(\bar{x}^{2}-\alpha^{2}\right)$ for $x \in \operatorname{Ker} L$. Therefore, on identifying $\operatorname{Ker} L$ with $\mathbb{R}$, the above degree equals $\pm \operatorname{deg}\left(g, I^{+}, 0\right)$, where $g(s)=s^{2}-\alpha^{2}(s \in \mathbb{R})$ and $I^{+}$is a small interval around $\alpha$. Finally, by definition of degree for $C^{1}$ maps,

$$
\operatorname{deg}\left(g, I^{+}, 0\right)=\operatorname{sign} g^{\prime}(\alpha)=\operatorname{sign} 2 \alpha=1
$$

Now apply Theorem 1 with $U=E, U^{\prime}=B^{+}$. This gives the existence of a connected subset $\Sigma$ of solutions of (3.1),

$$
\Sigma \dot{\subset}\{(\lambda, x): \lambda \neq 0\} \cup\left\{\left(0, x^{+}\right)\right\}
$$

which, due to a priori bounds in $\lambda$ and $x$ found before, must satisfy the third possibility in Theorem 1, and therefore necessarily contains $x^{-}$in its closure.

Next we prove that the solution set of (3.1) contains a loop around $\left(0, x^{+}\right)$. To be precise, we shall prove that if $C$ denotes the connected component of $G^{-1}(0)$ containing $\left(0, x^{+}\right)$then there exists a neighborhood $W$ of $\left(0, x^{+}\right)$such $C \backslash W$ is connected while $C \cap W \backslash\left\{\left(0, x^{+}\right)\right\}$is disconnected. To do this we shall follow the arguments given in $[4$, Prop. 4.4].

First observe that, on using the implicit function theorem, we can find an $\varepsilon>0$, a neighborhood $B_{0}$ of $x_{0}=x^{+}$(we can take $B_{0}=B^{+}$without loss of generality) and a continuous function $x=x(\lambda)$ defined on $(-\varepsilon, \varepsilon)$ with $x(0)=x_{0}$ such that

$$
G^{-1}(0) \cap B=\{(\lambda, x(\lambda)):-\varepsilon<\lambda<\varepsilon\},
$$

where $B=(-\varepsilon, \varepsilon) x B_{0}$. From part (c) of [3, Theorem 4.1], it follows easily that

$$
\Sigma \cap B=\{(\lambda, x(\lambda)):-\varepsilon<\lambda<\varepsilon\} .
$$

Now let $C^{ \pm}$be the two pieces of $C$ emanating at $( \pm \varepsilon, x( \pm \varepsilon))$. We claim that $C^{-} \cap C^{+}$is nonempty and hence $C \backslash B=C^{-} \cup C^{+}$, being the union of two intersecting connected subsets, is connected. Indeed suppose that $C^{-} \cap C^{+}=\varnothing$. Then, since $C^{-}$and $C^{+}$are compact, by a standard argument (see e.g. Whyburn [7]) it follows that there exist open 
disjoint bounded sets $V^{-}, V^{+}$such that $C^{ \pm} \subset V^{ \pm}$,

$$
0 \subset V=V^{-} \cup V^{+} \cup B
$$

and $G^{-1}(0) \cap \partial V=\varnothing$.

Now define a continuous map $g: V \rightarrow \mathbb{R}$ by

$$
g(\lambda, x)=\left\{\begin{aligned}
\lambda & \text { on } B \\
\pm \varepsilon & \text { on } V^{ \pm}
\end{aligned}\right.
$$

On using the excision, homotopy and product properties of the Leray-Schauder degree, we get the following chain of equalities:

$$
\begin{aligned}
\operatorname{deg}((G, g), V, 0) & =\operatorname{deg}((G, g), B, 0) \\
& =\operatorname{deg}\left(\left(G_{0}, g\right), B, 0\right) \\
& =\operatorname{deg}(g,(-\varepsilon, \varepsilon), 0) \operatorname{deg}\left(G_{0}, B_{0}, 0\right) \\
& =\operatorname{deg}\left(G_{0}, B_{0}, 0\right) .
\end{aligned}
$$

Therefore the first degree is nonzero; this implies, taking into account that $G^{-1}(0) \cap \partial V=\varnothing$, and the map $(G, g-2 \varepsilon)$ has nonzero degree in $V$, a contradiction since $|g(\lambda, x)| \leqq \varepsilon$ on $V$. Moreover $C \cap B$ is disconnected on removing the point $\left\{\left(0, x_{0}\right)\right\}$ by the continuity of the function $x=x(\lambda)$. Hence we can conclude that $G^{-1}(0)$ contains a subset which looks like a loop.

Acknowledgment. The author wishes to thank J. Mawhin and I. Massabò for helpful suggestions.

\section{REFERENCES}

1. M. Furi and M. P. Pera, Co-bifurcating branches of solutions for nonlinear eigenvalue problems in Banach spaces, Ann. Mat. Pura Appl. (4) 135 (1983), 119-131.

2. R. E. Gaines and J. L. Mawhin, Coincidence degree and nonlinear differential equations, Lecture Notes in Mathematics no. 568 (Springer, 1977).

3. J. Ize, I. Massabò, J. Pejsachowicz and A. Vignoli, Nonlinear multiparameteric equations: structure and topological dimension of global branches of solutions, Rapporto no. 6 (1983), Dipartimento di Matematica, Università della Calabria.

4. J. Ize, I. Massabò, J. Pejsachowicz and A. Vignoli, Structure and dimension of global branches of solutions to multiparametric nonlinear equations, to appear in the Proceedings of the AMS Conference held in Berkeley, July 1983.

5. N. G. Lloyd, Degree theory, Cambridge Tracts in Mathematics, 73 (Cambridge University Press, 1978).

6. M. Martelli, Large oscillations of forced nonlinear differential equations, Contemporary Mathematics 21, (Amer. Math. Soc., 1983).

7. G. T. Whyburn, Topological analysis (Princeton University Press, 1958).

Dipartimento di Matematica

Università della Calabria

87036 Arcavacata di Rende (CS) 